\title{
Accelerated generation of oligodendrocyte progenitor cells from human induced pluripotent stem cells by forced expression of Sox10 and Olig2
}

\author{
Pengyan $\mathrm{Li}^{1,2,3 \dagger}$, Mo Li ${ }^{1,2 \uparrow}$, Xihe Tang ${ }^{1,2}$, Shuyan Wang ${ }^{1,2}$, Y. Alex Zhang ${ }^{1,3^{*}}$ \& Zhiguo Chen ${ }^{1,2,3^{* *}}$ \\ ${ }^{1}$ Cell Therapy Center, Beijing Institute of Geriatrics, Xuanwu Hospital, Capital Medical University, and Key Laboratory of Neurodegeneration, \\ Ministry of Education, Beijing 100053, China; \\ ${ }^{2}$ Center of Neural Injury and Repair, Beijing Institute for Brain Disorders, Beijing 100069, China, \\ ${ }^{3}$ Center of Parkinson's Disease, Beijing Institute for Brain Disorders, Beijing 100069, China
}

Received June 8, 2016; accepted August 31,2016; published online October 25, 2016

\begin{abstract}
Oligodendrocyte progenitor cells (OPCs) hold great promise for treatment of dysmyelinating disorders, such as multiple sclerosis and cerebral palsy. Recent studies on generation of human OPCs mainly use human embryonic stem cells (hESCs) or neural stem cells (NSCs) as starter cell sources for the differentiation process. However, NSCs are restricted in availability and the present method for generation of oligodendrocytes (OLs) from ESCs often requires a lengthy period of time. Here, we demonstrated a protocol to efficiently derive OPCs from human induced pluripotent stem cells (hiPSCs) by forced expression of two transcription factors (2TFs), Sox10 and Olig2. With this method, PDGFR $\alpha^{+}$OPCs can be obtained in 14 days and $\mathrm{O}^{+}$OPCs in 56 days. Furthermore, OPCs may be able to differentiate to mature OLs that could ensheath axons when co-cultured with rat cortical neurons. The results have implications in the development of autologous cell therapies.
\end{abstract}

oligodendrocyte progenitors, iPSC, differentiation, demyelination

Citation: $\quad$ Li, P., Li, M., Tang, X., Wang, S., Zhang, Y.A., and Chen, Z. (2016). Accelerated generation of oligodendrocyte progenitor cells from human induced pluripotent stem cells by forced expression of Sox10 and Olig2. Sci China Life Sci 59, 1131-1138. doi: 10.1007/s11427-016-0165-3

\section{INTRODUCTION}

The loss of OLs and myelin in the central nervous system (CNS) often causes substantial morbidity and mortality, such as multiple sclerosis and leukodystrophies. Currently available treatments targeting the immune system are highly effective at reducing the intermittent episodes of inflammation, but they do not influence the progressive course of disease and the long-term prognosis is generally poor. OPCs can differentiate into OLs which ensheath axons with myelin during

$\dagger$ Contributed equally to this work

*Corresponding author (email: yaz@bjsap.org)

***Corresponding author (email: chenzhiguo@gmail.com) brain development and remyelinate axons after brain damage. Thus OPCs have been identified as a promising cell population for therapeutic approaches in demyelinating diseases. Many studies have been focused on differentiation of OPCs from ESCs (Cloutier et al., 2006; Kang et al., 2007; Piao et al., 2015) and NSCs (Hwang et al., 2009; Sher et al., 2009), or generation of OPCs by direct lineage conversion for the treatment of those diseases (Najm et al., 2013; Yang et al., 2013). However, there are considerable drawbacks to the actual application of these cells. Grafts of OPCs derived from hESCs are allogeneic and may require immunosuppressive treatment after transplantation, apart from the ethical concerns associated with hESCs. Human NSCs (hNSCs) exist at the subven- 
tricular zone (SVZ) of lateral ventricle wall in adult CNS, or can be isolated from fetal brain. However, either case means restricted availability.

Human iPSCs can be generated from many somatic cell types; thus patient-specific iPSCs could offer an autologous origin of OPCs. A recent paper suggests the feasibility of obtaining iPSCs-derived OPCs through an embryoid body (EB) stage; however, it takes about four months to get a large number of PDGFR $\alpha^{+}$OPCs (Wang et al., 2013). Recently, Fossati and Douvaras improved the differentiation protocol by using monolayer culture on Matrigel, together with small molecule treatment. In this way, generation of oligodendrocytes from iPSCs can be shortened from 95 days to 75 days (Douvaras and Fossati, 2015; Douvaras et al., 2014). We were inspired by the report that functional induced neurons can be generated from hiPSCs within two weeks by forced expression of a single transcription factor (Zhang et al., 2013). Sox10 and Olig2 play key roles in OL specification (Cahoy et al., 2008; Dugas et al., 2006; Goldman and Kuypers, 2015; Lu et al., 2000; Stolt et al., 2004; Wang et al., 2014; Zhou et al., 2000), and therefore were chosen in attempt to accelerate the generation of OPCs from hiPSCs.

In this study, we demonstrated a method of generating PDGFR $\alpha^{+}$pre-OPCs in less than two weeks, which could be further maturated into $\mathrm{O}^{+}$late-OPCs. In addition, we co-cultured induced OPCs (iOPCs) with rat cortical neurons to examine the capacity to ensheath axons.

\section{RESULTS}

\section{Generation of iOPCs within two weeks}

To accelerate the speed of OPC generation, we designed an experimental procedure (Figure 1A) to direct iPSCs into iOPCs by forced expression of Sox 10 and Olig2. Following infection of virus encocoding Sox 10 and Olig2 and selection with hygromycin, about $60 \%$ of cells were co-infected with the 2TFs (Sox10 and Olig2), as evidenced by immunostaining against Sox10 and Olig2 (data not shown). After infection with 2TFs and treated with OPC medium, a marked morphological change from typical iPSCs to bipolar cells was shown within two weeks (Figure 1B-E). A subpopulation of the iPSCs-derived cells could proliferate in response to OPC medium added with PDGF and stained positive for PDGFR $\alpha$ - a defined specific marker for OPC (Sim et al., 2011; Wang et al., 2013), which was not expressed by iPSCs (Figure 1F). At day 14, a large proportion of cells were labeled with PDGFR $\alpha$ owing to the combination of Sox 10 and Olig2 compared with negative control infected with iDuet101-GFP only (Figure 1G-I).

\section{Combination of Sox10 and Olig2 is better than either fac- tor alone in iOPC generation}

To examine whether a single transcription factor (Sox10 or
Olig2) is sufficient to promote the generation of iOPCs, we set up four experimental groups, (A) Sox10, (B) Olig2, (C) Sox10 and Olig2, and (D) negative controls (NC) without virus infection. At day 15, we observed OPC-like cells with a bipolar morphology (Figure 2A-D) and the iOPCs could proliferate in response to PDGF-AB and bFGF (Pang et al., 2013) from groups with viral infection. Cells from the four groups were stained for PDGFR $\alpha$. The results showed that the proportion of PDGFR $\alpha^{+}$cells from Sox10+Olig2 group was more than that of single factor groups, which was further higher than that of negative control group (Figure $2 \mathrm{E}-\mathrm{H}$, M). To investigate the differential capacity of iOPCs, cells were exposed to differential condition containing T3, NT3, IGF-1 (known to be beneficial for oligodendrocyte differentiation or survival) (Douvaras and Fossati, 2015; Najm et al., 2013; Pang et al., 2013). Cells were stained with O4 antibodies (Douvaras and Fossati, 2015; Pang et al., 2013; Yang et al., 2013) every other week to test whether induced oligodendrocytes (iOLs) had appeared. At the fourth week of differentiation, around $42 \%$ of cells were O4-positive in Sox 10+Olig2 group, higher than that of single factor groups and the negative group (Figure 2I-L, N).

The endogeneous gene expression was examined by using RT-PCR. Along the course of differentiation, iPSC-related genes OCT4, NANOG, and SOX2 were shut down, and OPCand OL-related genes were turned on (Figure 3A-D), confirming that the cells in the three groups had committed to the oligodendrocyte lineage at the molecular level. The exogenous gene expression was also monitored, which was still detectable at day 15 , but the transgenes were gradually silenced afterwards (Figure 3E).

To further confirm the generation of iOPCs and iOLs from the Sox10+Olig2 group, we stained the cells with additional markers of OPC and OL such as NKX2.2, A2B5 (Wang et al., 2013; Yang et al., 2013), O1 and CNPase, using the experimental procedure described in Figure 1A. Then we observed proliferative cells in response to growth factors with bipolar, OPC-like morphologies (Figure 4A). Cells were stained with NKX2.2, A2B5, PDGFR $\alpha$ and NG2 (specific markers of OPCs) in an attempt to verify the generation of iOPCs (Figure 4C-E). The proportion of $\mathrm{A} 2 \mathrm{~B} 5^{+} / \mathrm{PDGFR}^{+}$ double positive cells was about $18 \%$ at day 14 . Also, $\mathrm{GFAP}^{+}$ astrocytes could be generated during the differentiation (Figure 4F). After eight weeks of differentiation, many processes took shape in induced cells (Figure 4B), and the cells were positive for O1, CNPase and O4 (markers of OL lineage) (Figure 4G-J), suggesting that OLs might have been differentiated from hiPSCs by forced expression of 2TFs (Sox10 and Olig2).

\section{iOPCs may generate iOLs when co-cultured with cortical neurons in vitro}

To evaluate the myelinating function of iOLs obtained from 

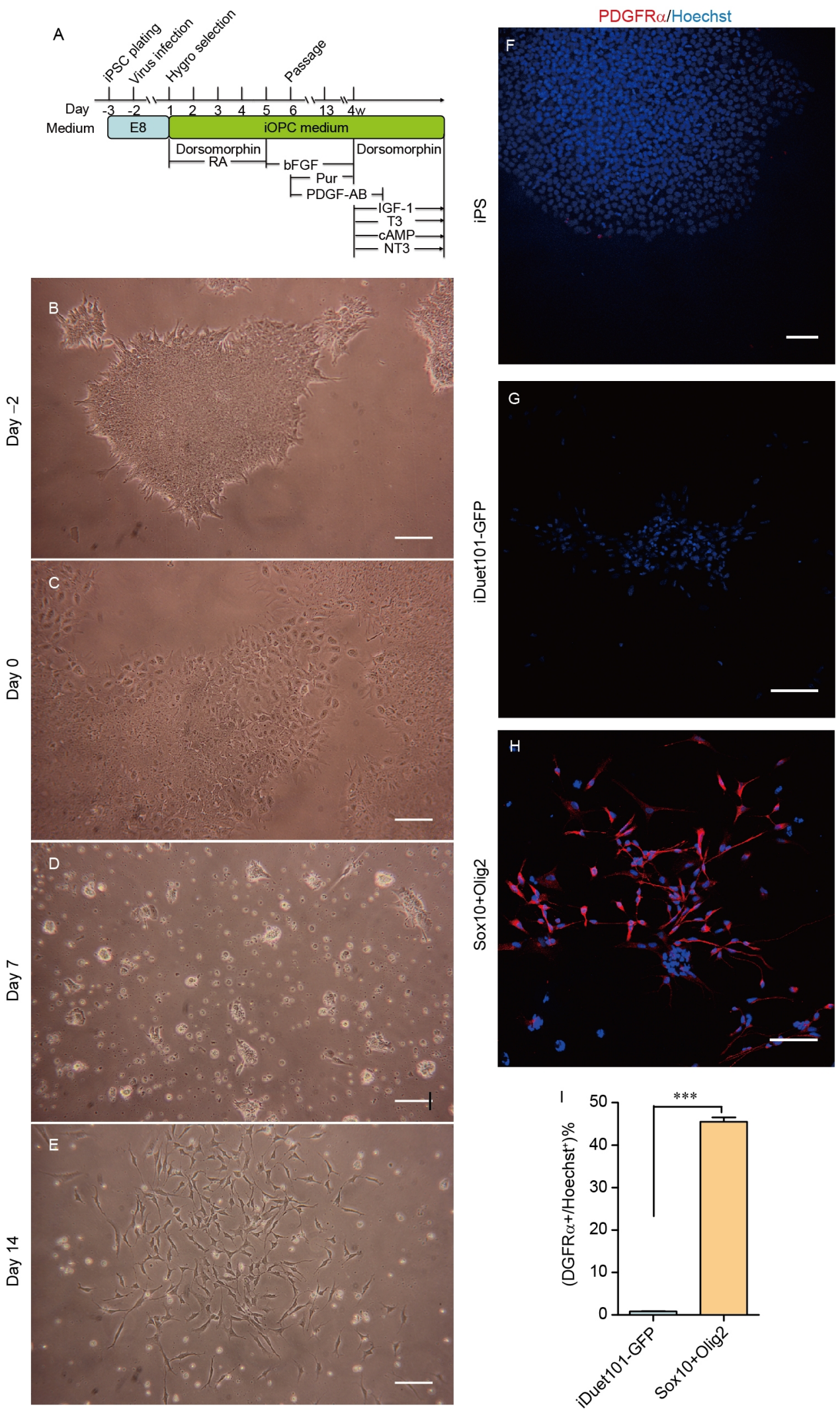

Figure 1 (Color online) Acceralted generation of iOPCs. A, Diagram of iOPC generation. B-E, Morphology changes from iPSCs to iOPCs by infection with 2TFs. B, hiPSCs before adding virus. C, hiPSCs infected with virus for $36 \mathrm{~h}$. D, cells passaged on PDL/LMN. E, morphology of iOPCs at day 14 . F, iPSCs stained negative for PDGFR $\alpha$. G, iPSCs infected with iDuet101-GFP at differentiation day 14 were negative for PDGFR $\alpha$. H, iPSCs infected with Sox10 and Olig2 at differentiation day 14. I, the proportion of PDGFR $\alpha$-positive cells in Sox10+Olig2 group was significantly higher than that of control group at day 14. ***, $P<0.001$; Blue in F-H, Hoechst; Red in F-H, PDGFR $\alpha$; Scale bars, $200 \mu \mathrm{m}(\mathrm{B}-\mathrm{E}), 100 \mu \mathrm{m}(\mathrm{F})$, and $75 \mu \mathrm{m}(\mathrm{G}$ and $\mathrm{H})$. TF, transcription factor. 

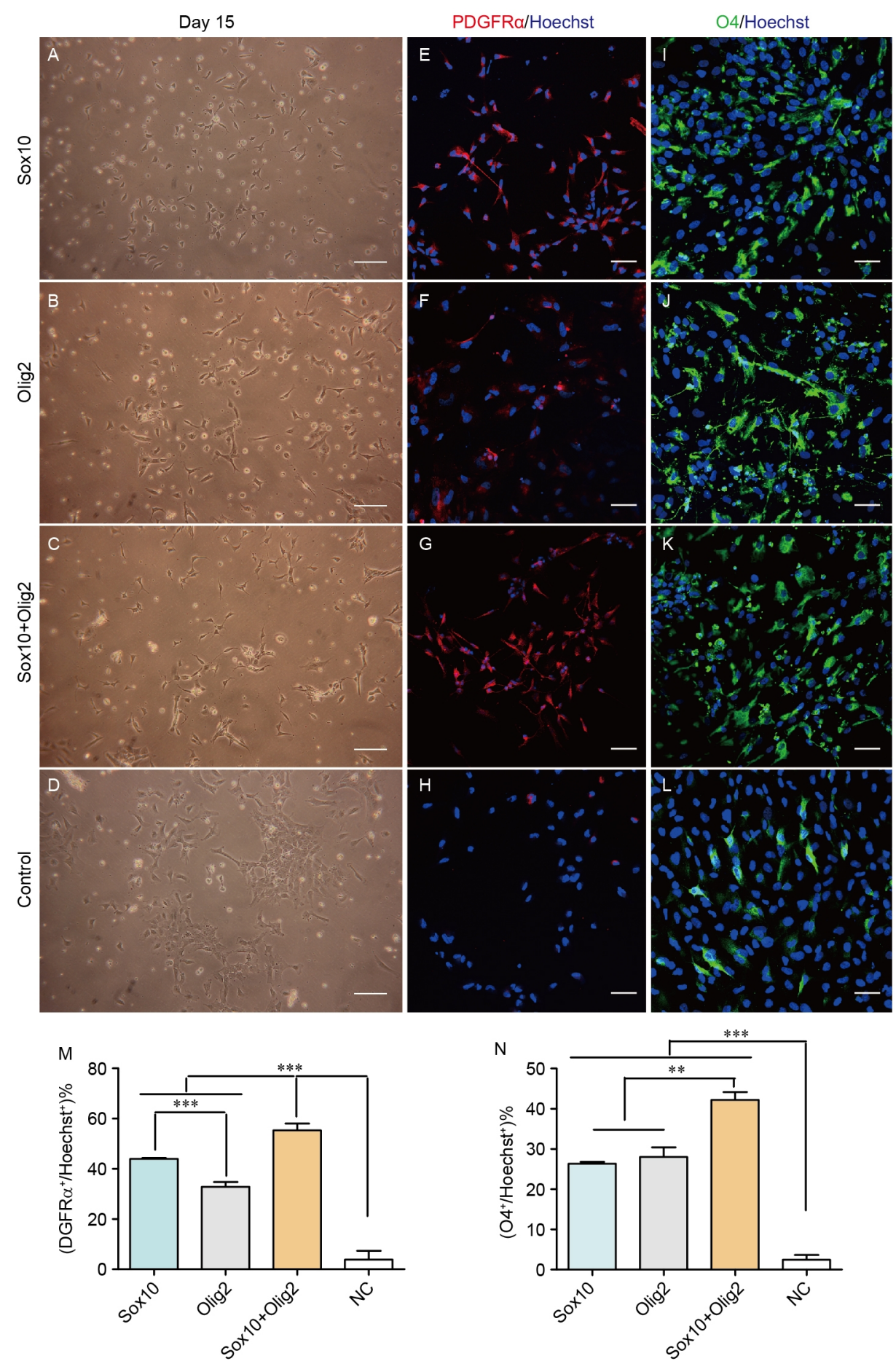

Figure 2 (Color online) Efficiency of iOPC generation in different groups. A-D, The morphology of cells from the four groups at day 15 . Sox 10 (A), Olig2 (B), Sox10+Olig2 (C), none-virus control (D), Scale bars, $200 \mu \mathrm{m}$. E-N, The proportions of PDGFR $\alpha-$ and O4-positive cells in Sox10+Olig2 group were significantly higher than those of other groups at day 15 and 56. E-L, Sox10 (E and I), Olig2 (F and J), Sox 10+Olig2 (G and K), none-virus control (H and L). PDGFR $\alpha$ (red), O4 (green), Hoechst (blue), Scale bars, $50 \mu \mathrm{m}$. (M and N) two tailed $t$ test; ${ }^{* * *}, P<0.001 ; * *, P<0.01$.

hiPSCs, induced cells were co-cultured with rat cortical neurons isolated at embryonic day 17 (E17). Cortical neurons (about $4 \times 10^{5}$ ) were seeded on coverslips coated with PDL/Lamnin and were treated with Ara-c for four days. Meanwhile, iOPCs were infected with iDuet101-GFP at day 21 of differentiation. Then GFP-expressing iOPCs (about
$1 \times 10^{5}$ ) were plated onto cortical neurons and cultured in differentiation medium. Cells were fixed and stained at day 7 and 14 after co-culture. At day 7 in co-culture, some seeded cells $\left(\mathrm{GFP}^{+}\right)$showed morphology with complex processes and stained positive for the OL marker O4 (Figure 5A-C). At day 7 in co-culture, the proportion of O4-positive iOLs 


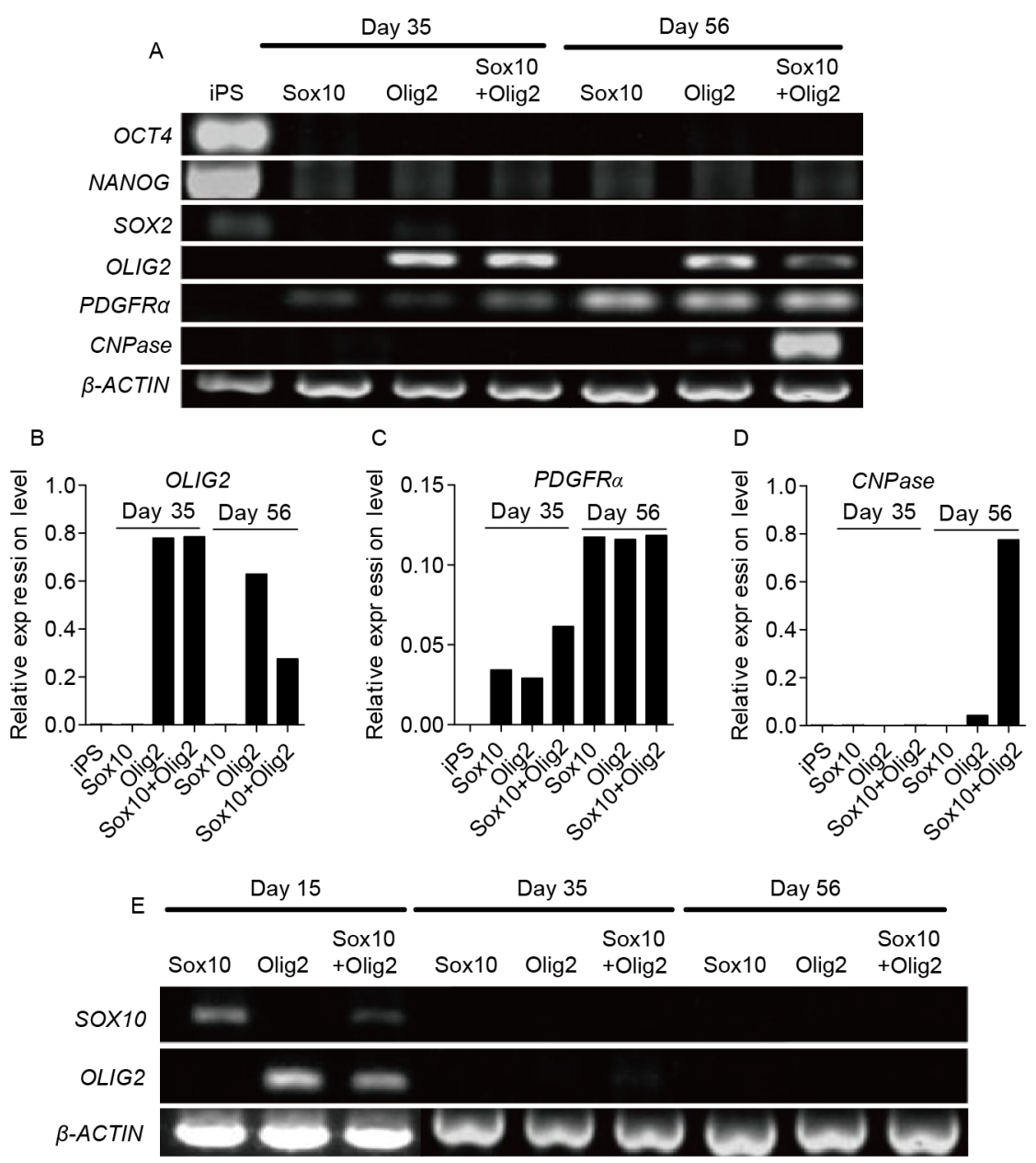

Figure 3 RT-PCR analysis of endogenous and exogenous factor expression. A, RT-PCR analysis of endogenous expression of iPSC-related genes OCT4/NANOG/SOX2, and OPC-related genes OLIG2/PDGFRA, and OL-related gene CNPase. B-D, Relative expression levels of OLIG2 (B), PDGFR (C) and CNPase (D) normalized with $\beta$-ACTIN expression. E, RT-PCR analysis of exogenous factor expression (SOX10 and OILG2) in the three experimental groups.

was about $5 \%$, and the ratio of OLs with typical morphology (with arborized processes) to those with aberrant morphology (flattened with few processes) was about 1:2. Compared with direct differentiation from iOPCs without co-culture, co-culture with cortical neurons may have sped up the generation of iOLs. At day 14 in co-culture, around $0.5 \%$ of rat cortical neurons (Tuj- $1^{+}$and $\mathrm{GFP}^{-}$) had axons co-labeled with the processes of iOLs $\left(\mathrm{GFP}^{+}\right)$(Figure 5D). The results indicate that the obtained iOLs using two factors might possess the capacity to myelinate axons.

\section{DISCUSSION}

In this study, we described a protocol to convert hiPSCs into OPCs by forced expression of two transcription factors, Sox 10 and Olig2. Introduction of these two factors markedly accelerated the generation of OPCs and the efficiency was higher with two combined factors than that with each individual factor.

A recent study published by Wernig's group (Yang et al.,
2013) reported that three factors, Sox10, Olig2, and Zfp536 are sufficient to reprogram rodent fibroblasts into OPCs. In another study published from Tesar's lab (Najm et al., 2013), it was shown that Sox10, Olig2, and Nkx6.2 are capable of converting mouse fibroblasts into OPCs. In both studies, Sox10 and Olig2 were included in the three factor combination, suggesting their central roles in OPC development and maintainence. Different cell types in a way reflect a certain functional state governed by a network of factors/proteins. A master factor may reset the network by feed-forward and feed-back signaling pathways and therefore lead to a different functional state/cell type. Inspired by the finding that a single factor can signigicantly accelerate neuronal induction from ES cells, we reasoned that Sox10 and Olig2 may also speed up the conversion of iPSCs into OPCs, given the key functions of these two factors. Indeed, in the current study, we found that Sox 10 and Olig2 can reduce the otherwise lengthy differentiation process to two weeks. However, it is still unclear whether the OPCs obtained this way are the same as the primary OPCs and/or ESC-derived OPCs through EB stage. 

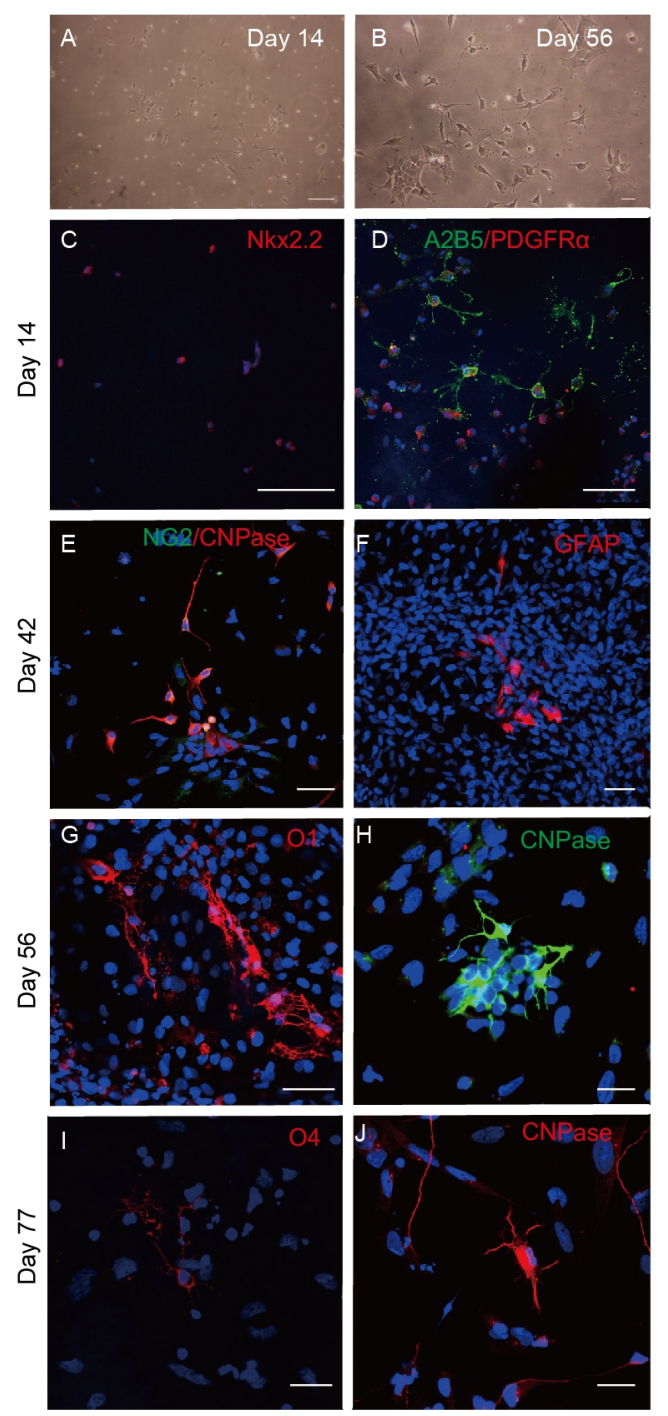

Figure 4 (Color online) Generation of iOLs from hiPSCs. A and B, The morphology of iOPCs at day 14 (A) and day 56 (B). C, Nkx2.2 (red). D, merged staining results for A2B5 (green) and PDGFR $\alpha$ (red). E, merged staining results for NG2 (green) and CNPase (red). F, GFAP (red). G, O1 (red). H, CNPase (green). I, O4 (red). J, CNPase (red), Hoechst (blue). Scale bars, $200 \mu \mathrm{m}$ (A), $100 \mu \mathrm{m}$ (B and C), $75 \mu \mathrm{m}$ (D), $50 \mu \mathrm{m}$ (E-G), $25 \mu \mathrm{m}$ (H-J).

Further studies applying gene expression profiling and in vivo transplantation in demyelination models are needed to address these questions.

\section{MATERIALS AND METHODS}

\section{Vector construction}

The coding regions of Sox 10 and Olig2 were amplified by PCR using human cDNA as templates, and then cloned into Kpn I/BsrG I site of the iDuet101 plasmid, respectively (Zhou et al., 2007). The constructed vectors were transformed into E. coli DH5 $\alpha$ for expansion followed by extraction and sequence confirmation.

\section{Production of lentivirus}

Lentiviruses were produced according to a previously published study (Sheng et al., 2012). Briefly, HEK293T cells were cultured in OPTi-MEM supplemented with VSVG, delt 8.74, recombiant plasmid and lipo2000. After $8 \mathrm{~h}$, OPTiMEM was replaced with 293 T medium consisting of DMEM, FBS, PS and Glutamax. Three days later, supernatant containing lentivirus was harvested and concentrated by centrifugation after filtering with $0.45 \mu \mathrm{m}$ PVDF membrane.

\section{Generation of iOPCs from human iPSCs}

Human iPSCs, a gift from our colleague in our own lab (Wang et al., 2015), were plated in matrigel-coated six-well-culture plate and cultured in E8 medium (special medium for iPSCs). Upon about $30 \%$ confluence, media containning lentivirus and $4 \mu \mathrm{g} \mathrm{mL}^{-1}$ polybrene were mixed and added freshly onto hiPSCs. Viral medium was changed after $36 \mathrm{~h}$ and replaced with E8 medium to assist the recovery of hiPSCs (Figure 1C). The day when the virus was discarded was termed "Day 0". iDuet101 was contructed to include a Hygro gene, which can be used for selection with hygromycin starting on Day 1. On Day 1, E8 medium was replaced with iOPCs basic medium consisting of DMEM/F12, N2, B27 without vitamin A, and glutamax, supplemented with $3 \mu \mathrm{mol} \mathrm{L} \mathrm{L}^{-1}$ dorsomorphin and $1 \mu \mathrm{mol} \mathrm{L}{ }^{-1}$ RA (Jiang et al., 2010) for five days. When the cells of negative control without virus infection all died by hygromycin treatment, hygromycin was removed from the experimental group. At day 6, cells were transferred onto coverslips coated with poly-D-lycin(PDL)-laminin by treatment with accutase. As is shown in the flow diagram (Figure 1A), the following molecules were used: $20 \mathrm{ng} \mathrm{mL}^{-1}$ platelet-derived growth factor-AB (PDGF-AB), $20 \mathrm{ng} \mathrm{mL}{ }^{-1}$ basic fibroblast growth factor (bFGF), $60 \mathrm{ng} \mathrm{mL} \mathrm{mL}^{-1}$ thriiodo-L-thytonine (T3), $10 \mathrm{ng} \mathrm{mL}^{-1}$ neurotrophin 3 (NT3), $1 \mu \mathrm{mol} \mathrm{L}^{-1}$ purmophamine (Pur), $10 \mathrm{ng} \mathrm{mL}^{-1}$ insulin-like growth factor-1 (IGF-1) and $1 \mu \mathrm{mol} \mathrm{L}{ }^{-1} \mathrm{cAMP}$.

\section{Immunofluorescence}

Cells were washed twice in PBS and fixed with 4\% paraformaldehyde (PFA) for $15 \mathrm{~min}$ at room temperature (RT). Then cells were immersed in blocking buffer consisting of $0.3 \%$ Triton X-100 and 3\% donkey serum for at least $1 \mathrm{~h}$, then incubated with primary antibodies overnight at $4{ }^{\circ} \mathrm{C}$. The following primary antibodies were used: rabbit anti-PDGFR $\alpha$ (Santa Cruz, USA, 1:200), rabbit anti-CNPase (Multi Science, USA, 1:200), rabbit anti-NKX2.2 (Santa Cruz, 1:200), rabbit anti-GFAP (Beijing Zhong Shan-Golden Bridge, 1:100), rabbit anti-Olig2 (Millipore, USA, 1:500), mouse anti-NG2 (Abcam, USA, 1:100), mouse anti-A2B5 (Abcam, 1:800), mouse anti-O1 (eBioscience, USA, 1:100), mouse anti-O4 (R\&D Systems, USA, 1:400), mouse anti-Tuj-1(Millipore, 1:400), goat anti-Sox10 (Santa Cruz, 1:50). Next, cells were incubated with secondary anti- 

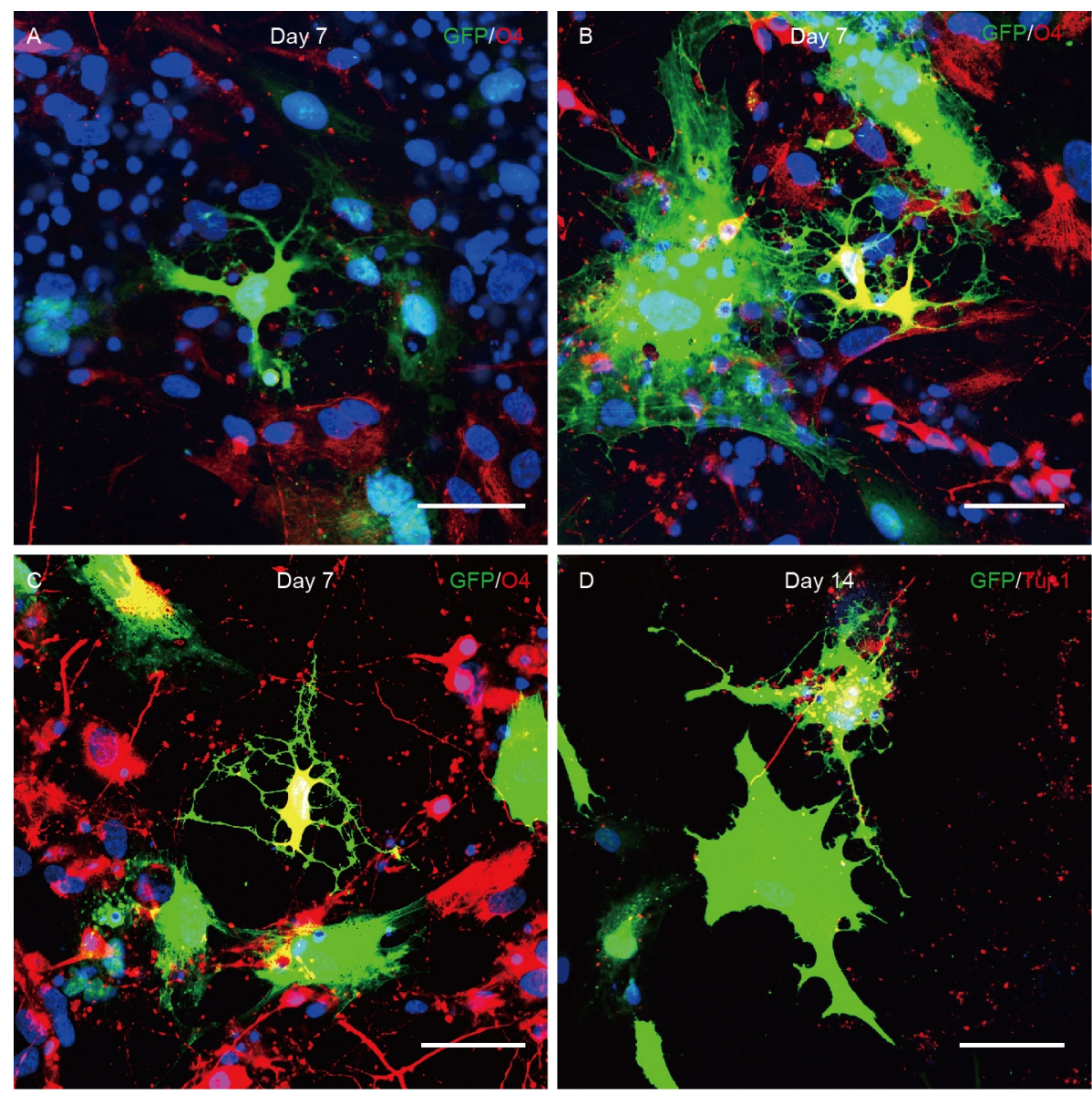

Figure 5 (Color online) iOPCs co-cultured with rat cortical neurons. A-C, co-culture at day 7, merged staining results for GFP (green) and O4 (red). D, co-culture at day 14, merged staining results for GFP (green) and Tuj-1 (red). Scale bars, $50 \mu \mathrm{m}$ (A and B), $25 \mu \mathrm{m}$ (C), $75 \mu \mathrm{m}$ (D).

bodies for $2 \mathrm{~h}$ at RT. Omission of primary antibodies served as negative controls.

Compliance and ethics The author(s) declare that they have no conflict of interest.

Acknowledgements This work was supported by the Stem Cell and Translation National Key Project (2016ZY05002449), National Basic Research Program of China (2011CB965103, 2012CBA01307), National Natural Science Foundation of China (81661130160, 81422014, 81561138004), Beijing Municipal Natural Science Foundation (5142005), and Royal Society-Newton Advanced Fellowship (NA150482).

Cahoy, J.D., Emery, B., Kaushal, A., Foo, L.C., Zamanian, J.L., Christopherson, K.S., Xing, Y., Lubischer, J.L., Krieg, P.A., Krupenko, S.A., Thompson, W.J., and Barres, B.A. (2008). A transcriptome database for astrocytes, neurons, and oligodendrocytes: a new resource for understanding brain development and function. J Neurosci 28, 264-278.

Cloutier, F., Siegenthaler, M.M., Nistor, G., and Keirstead, H.S. (2006). Transplantation of human embryonic stem cell-derived oligodendrocyte progenitors into rat spinal cord injuries does not cause harm. Regen Med $1,469-479$.

Douvaras, P., and Fossati, V. (2015). Generation and isolation of oligodendrocyte progenitor cells from human pluripotent stem cells. Nat Protoc $10,1143-1154$

Douvaras, P., Wang, J., Zimmer, M., Hanchuk, S., O’Bara, M.A., Sadiq, S.,
Sim, F.J., Goldman, J., and Fossati, V. (2014). Efficient generation of myelinating oligodendrocytes from primary progressive multiple sclerosis patients by induced pluripotent stem cells. Stem Cell Rep 3, 250-259.

Dugas, J.C., Tai, Y.C., Speed, T.P., Ngai, J., and Barres, B.A. (2006). Functional genomic analysis of oligodendrocyte differentiation. J Neurosci 26, 10967-10983.

Goldman, S.A., and Kuypers, N.J. (2015). How to make an oligodendrocyte. Development 142, 3983-3995.

Hwang, D.H., Kim, B.G., Kim, E.J., Lee, S.I., Joo, I.S., Suh-Kim, H., Sohn, S., and Kim, S.U. (2009). Transplantation of human neural stem cells transduced with Olig2 transcription factor improves locomotor recovery and enhances myelination in the white matter of rat spinal cord following contusive injury. BMC Neurosci 10, 117.

Jiang, P., Selvaraj, V., and Deng, W. (2010). Differentiation of embryonic stem cells into oligodendrocyte precursors. J Vis Exp doi: 103791/1960.

Kang, S.M., Cho, M.S., Seo, H., Yoon, C.J., Oh, S.K., Choi, Y.M., and Kim, D.W. (2007). Efficient induction of oligodendrocytes from human embryonic stem cells. Stem Cells 25, 419-424.

Lu, Q.R., Yuk, D., Alberta, J.A., Zhu, Z., Pawlitzky, I., Chan, J., McMahon, A.P., Stiles, C.D., and Rowitch, D.H. (2000). Sonic hedgehog-regulated oligodendrocyte lineage genes encoding bHLH proteins in the mammalian central nervous system. Neuron 25, 317-329.

Najm, F.J., Lager, A.M., Zaremba, A., Wyatt, K., Caprariello, A.V., Factor, D.C., Karl, R.T., Maeda, T., Miller, R.H., and Tesar, P.J. (2013). Transcription factor-mediated reprogramming of fibroblasts to expandable, myelinogenic oligodendrocyte progenitor cells. Nat Biotechnol 31, 426-433.

Pang, Y., Fan, L.W., Tien, L.T., Dai, X.M., Zheng, B., Cai, Z., Lin, R.C.S., and Bhatt, A. (2013). Differential roles of astrocyte and microglia in 
supporting oligodendrocyte development and myelination in vitro. Brain Behav 3, 503-514.

Piao, J., Major, T., Auyeung, G., Policarpio, E., Menon, J., Droms, L., Gutin, P., Uryu, K., Tchieu, J., Soulet, D., and Tabar, V. (2015). Human embryonic stem cell-derived oligodendrocyte progenitors remyelinate the brain and rescue behavioral deficits following radiation. Cell Stem Cell $16,198-210$

Sheng, C., Zheng, Q., Wu, J., Xu, Z., Wang, L., Li, W., Zhang, H., Zhao, X.Y., Liu, L., Wang, Z., Guo, C., Wu, H.J., Liu, Z., Wang, L., He, S., Wang, X.J., Chen, Z., and Zhou, Q. (2012). Direct reprogramming of Sertoli cells into multipotent neural stem cells by defined factors. Cell Res 22, 208-218.

Sher, F., van Dam, G., Boddeke, E., and Copray, S. (2009). Bioluminescence imaging of Olig2-neural stem cells reveals improved engraftment in a demyelination mouse model. Stem Cells 27, 1582-1591.

Sim, F.J., McClain, C.R., Schanz, S.J., Protack, T.L., Windrem, M.S., and Goldman, S.A. (2011). CD140a identifies a population of highly myelinogenic, migration-competent and efficiently engrafting human oligodendrocyte progenitor cells. Nat Biotechnol 29, 934-941.

Stolt, C.C., Lommes, P., Friedrich, R.P., and Wegner, M. (2004). Transcription factors Sox8 and Sox 10 perform non-equivalent roles during oligodendrocyte development despite functional redundancy. Development $131,2349-2358$.

Wang, J., Pol, S.U., Haberman, A.K., Wang, C., O’Bara, M.A., and Sim, F.J. (2014). Transcription factor induction of human oligodendrocyte progenitor fate and differentiation. Proc Natl Acad Sci USA 111,

\section{E2885-E2894.}

Wang, S., Bates, J., Li, X., Schanz, S., Chandler-Militello, D., Levine, C., Maherali, N., Studer, L., Hochedlinger, K., Windrem, M., and Goldman, S.A. (2013). Human iPSC-derived oligodendrocyte progenitor cells can myelinate and rescue a mouse model of congenital hypomyelination. Cell Stem Cell 12, 252-264.

Wang, S., Wang, B., Pan, N., Fu, L., Wang, C., Song, G., An, J., Liu, Z., Zhu, W., Guan, Y., Xu, Z.Q.D., Chan, P., Chen, Z., and Zhang, Y.A. (2015). Differentiation of human induced pluripotent stem cells to mature functional Purkinje neurons. Sci Rep 5, 9232.

Yang, N., Zuchero, J.B., Ahlenius, H., Marro, S., Ng, Y.H., Vierbuchen, T., Hawkins, J.S., Geissler, R., Barres, B.A., and Wernig, M. (2013). Generation of oligodendroglial cells by direct lineage conversion. Nat Biotechnol 31, 434-439.

Zhang, Y., Pak, C.H., Han, Y., Ahlenius, H., Zhang, Z., Chanda, S., Marro, S., Patzke, C., Acuna, C., Covy, J., Xu, W., Yang, N., Danko, T., Chen, L., Wernig, M., and Südhof, T.C. (2013). Rapid single-step induction of functional neurons from human pluripotent stem cells. Neuron 78, 785-798.

Zhou, B.Y., Ye, Z., Chen, G., Gao, Z.P., Zhang, Y.A., and Cheng, L. (2007). Inducible and reversible transgene expression in human stem cells after efficient and stable gene transfer. Stem Cells 25, 779-789.

Zhou, Q., Wang, S., and Anderson, D.J. (2000). Identification of a novel family of oligodendrocyte lineage-specific basic helix-loop-helix transcription factors. Neuron 25, 331-343.

Open Access This article is distributed under the terms of the Creative Commons Attribution License which permits any use, distribution, and reproduction in any medium, provided the original author(s) and source are credited. 